\title{
Diversity of flora used for the cure of equine diseases in selected peri-urban areas of Punjab, Pakistan
}

\author{
Khurram Goraya ${ }^{1}$, Zafar lqbal ${ }^{1 *}$, Muhammad Sohail Sajid ${ }^{1}$, Ghulam Muhammad², Qurat ul Ain ${ }^{1}$ and Muhammad Saleem ${ }^{3}$
}

\begin{abstract}
Background: Plants have widely been used and documented for their therapeutic potential in many parts of the world. There are, however, few reports on the use of plants for the treatment of diseases of equines. To this end, participatory epidemiology and rapid rural appraisal techniques were used to document the plants having pharmacotherapeutic significance against different ailments of equines in selected population of Punjab, Pakistan.

Methods: A survey was conducted to interview a total of 450 respondents (150 from each of the districts of Faisalabad, Lahore and Sargodha of Pakistan) to collect information about disease recognition of the equines and their treatment on a well - structured questionnaire. A total of 60 plants belonging to 40 families were documented. An inventory was developed depicting detailed information of plants used in treatment of different conditions of equines.
\end{abstract}

Results: The top ten species of plants used were: Allium cepa, Zingiber officinale, Vernonia anthelmintica, Capsicum annum, Brassica campestris, Trachyspermum ammi, Anethum graveolens, Picrorhiza kurroa, Azadirachta indica, and Citrullus colocynthis. Seeds were the most frequently used $(n=16 / 60)$ parts, followed by leaves $(n=12 / 60)$ and fruits $(n=11 / 60)$ of plants. Based on the combination of different parts of plants used in different ratios and variation in their dose or mode of preparation led to a large number of recipes/remedies against wounds, lameness, bronchitis, colic, anorexia, dermatitis, weakness, parasitism (internal \& external), fever, heat stress, urine retention, swelling, toxemia, and indigestion.

Conclusions: This study generated lot of data on phytomedicinal approach for the treatment of ailments in the equines in some selected areas. It would, therefore, be imperative to expand similar studies in other parts of Pakistan and elsewhere. Moreover, use of the documented plants may be validated employing standard scientific procedures, which may have their application in the drug discovery/development by the pharmaceutical industry.

Keywords: Phytotherapy, Plants, Equines, Indigenous, Ethnobotanicals, Punjab, Pakistan

\section{Background}

Equines (horses, donkeys and mules) are playing key roles in providing an economical draught power to resource-poor countries like Pakistan. Equines suffer from a variety of health conditions that not only hamper optimum performance, but also cause huge losses due to mortality [1]. Parasitism has been reported as the major health issue of equines in Punjab, Pakistan followed

\footnotetext{
*Correspondence: zafaruaf1@gmail.com

'Department of Parasitology, University of Agriculture, Faisalabad 38040 Punjab, Pakistan

Full list of author information is available at the end of the article
}

by wound, bacterial infections, lameness, bronchitis, dermatitis, and colic [2]. In addition to allopathic/ modern medicine, there is extensive use of traditional herbs for the treatment of different diseases in equines all over the world [3]. Ethnobotany has revealed that the indigenous knowledge of a community is a key player in the identification of medicinal plants which have been tested through generations in the human history [4]. Traditional medicine and bio-prospecting [5] may often lead to the development of a new herbal product based on their use by significant numbers of people over the extended periods of time [6]. The plant-based

\section{Biomed Central}


medicines have particularly been found promising as anti-parasitics, stomachics, and in treatment of various respiratory ailments [7-12]. So far, only a handful of investigations are reported on the use of plants for different ailments/conditions; however, inventories of plants for phyotherapy in the food animals are extensively reported. The present study was, therefore, carried out to document the plants being used in traditional veterinary practices for equines in some selected peri-urban areas of Punjab (Pakistan) where equines are frequently used for different purposes.

\section{Methods}

\section{Study districts}

Three districts of the central Punjab; Faisalabad, Lahore and Sargodha were included in the present survey. District Lahore is the capital of Punjab (second largest city of Pakistan after Karachi), while district Faisalabad is the hub of textiles (third largest city) of the country. District Sargodha is comparatively smaller city and considered as an agricultural trade center with various industries. The equine population of the three districts has been estimated as 24628 horses, 174994 donkeys and 7849 mules [13]. The use of equines in the three selected industrial districts of Punjab is frequent because it is the cheapest source for carriage of industrial raw materials and products from and to the market [2]. Figure 1 shows physical map of Punjab province and the three study districts.

\section{Selection of respondents}

A small scaled rapid rural appraisal (RRA), an exploratory phase [14] was conducted in metropolitan Faisalabad, Punjab, Pakistan for the purpose of collecting an initial

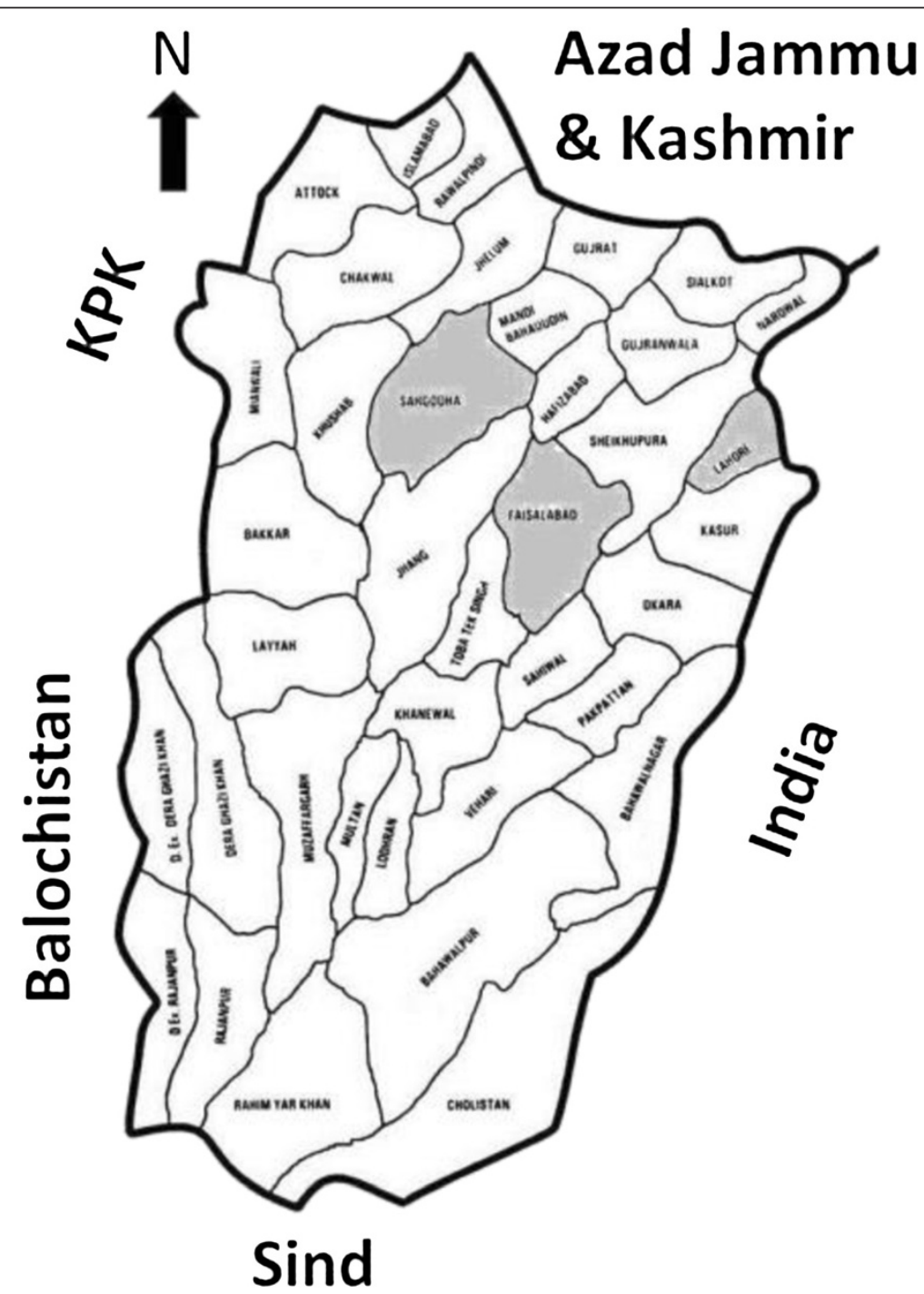

Figure 1 Physical map of the Punjab province, Pakistan indicating districts Faisalabad, Sargodha and Lahore. 
data from candidates who could participate in the second (surveillance) phase of the project.

Of the total 1000 registered farmers (who submitted their willingness to participate in the survey), 450 were selected as key respondents for this study. One hundred and fifty respondents represented each of the three districts of Faisalabad, Lahore and Sargodha. Selection of respondents within each district was done using proportional allocation and map grid methods in order to collect information from the selected districts. The selected respondents belonged to 9 sites each of districts Faisalabad and Sargodha, and 6 sites of Lahore (Table 1).

\section{Participatory epidemiology and collection of data}

A questionnaire containing a blend of open ended and closed (dichotomous and multiple choice) questions was prepared and refined through formal and informal testing [15]. For effective communication and data recording, a survey team was appointed; comprising of a veterinarian, professionals of The Brooke Hospital for Animals, and a community leader from the local village. Interviews, focused group discussions, and field visits were conducted with the respondents. The information about disease recognition of the equines and their treatments was collected using the well-structured questionnaire, open-ended interviews and guided-dialogue techniques. Focused group discussions were arranged to cross-check/ verify the information provided by the respondents to reach more accurate results. The respondents were asked to tell how they acquired the knowledge of phytotherapy related to the disease/condition recognition of equines. In addition, the direct observation approach was also followed as described by Etkins [16]. Thus, local names of plants, dose, parts of plant used, methods of preparation, and mode of administration were recorded. The farmers were asked to show the plant species described for the treatment of diseases/conditions for their taxonomic identification by the botanists at the Department of Botany, University of Agriculture, Faisalabad, Pakistan and the voucher specimens were preserved for record. The information collected was maintained in Microsoft Excel for further analyses and interpretation.

\section{Results}

\section{Respondents}

Equine owners/traditional veterinary healers were well familiar with the signs and symptoms of the diseases/ conditions of equines in the study area and a majority of the botanical ingredients used in treatment were of indigenous origin. Therefore, farmers had these remedies available at their door step or at the most in the nearby grocery shops. The respondents were not trained by any authority about usage of plants for treatment of their animals. In fact, their knowledge was based on folk beliefs and previous practices based on hit and trial methods.

\section{Diversity of plants used}

A total of 60 plants were documented (Table 2) for their use in different diseases/conditions of equines. Documented plants represented 40 families. Fabaceae was the largest represented family including five plants, followed by other families (Table 2). Composition, dosage, mode of preparation and administration, and frequency of usage of Traditional Veterinary Practices (TVPs) has been presented in Table 3. Plants and diversity of their usage in different diseases have been presented in Table 4 . Thirty one species were used for the treatment of multiple diseases/conditions. Piyaz (Onion; Allium cepa), Adrak (Ginger; Zingiber officinale), Kali zeeri (Iron weed; Vernonia anthelmintica), Mirch (Chilli; Capsicum annum), Sarsoon (Rapeseed plany; Brassica campestris), Ajwain (Carom seeds; Trachyspermum ammi), Ajwain (Dill; Anethum graveolens), Kutka (Picrorhiza kurroa), Neem (Azadirachta indica), and Kor tumma (Bitter gourd; Citrullus colocynthis) were the top ten most frequently used plants as part of prescriptions in different diseases/conditions of equines.

\section{Conditions reported against plant usage}

Number and nature of TVPs documented for the treatment of different diseases/conditions have been summarized in the Table 2. It is evident from the data (Table 4) that maximum number of plant based remedies/prescriptions was documented for the treatment of wounds $(n=57)$ followed by lameness $(n=40)$, bronchitis and colic $(n=21)$, anorexia $(n=19)$, dermatitis $(n=16)$, weakness $(n=13)$, internal parasites $(n=12)$, external parasites

Table 1 List of district wise sites for selection of respondents

\begin{tabular}{|c|c|c|c|c|c|c|c|c|c|}
\hline Districts/sites & 1 & 2 & 3 & 4 & 5 & 6 & 7 & 8 & 9 \\
\hline Faisalabad & Sidhupura & Chak 79 & $\begin{array}{l}\text { Ahmad } \\
\text { Nagar }\end{array}$ & Pansera & Chakera & Naitheri wala & Aziz Town & Karad Wala & $\begin{array}{l}\text { Brooke Hospital } \\
\text { for Animal Static } \\
\text { Clinic (UAF) }\end{array}$ \\
\hline Sargodha & Noor colony & Chak 87 & $\begin{array}{l}\text { Chak } 88 \\
\text { South }\end{array}$ & $\begin{array}{l}\text { Chak } \\
34\end{array}$ & $\begin{array}{l}\text { Fatima Jinnah } \\
\text { Road }\end{array}$ & $\begin{array}{l}\text { Farooq } \\
\text { Colony }\end{array}$ & $\begin{array}{l}\text { Makam-e- } \\
\text { Hayat }\end{array}$ & $\begin{array}{l}\text { Chak } 88 \\
\text { North }\end{array}$ & Saido wanan \\
\hline Lahore & $\begin{array}{l}\text { Shahdra } \\
\text { Town }\end{array}$ & $\begin{array}{l}\text { Fazal } \\
\text { Park }\end{array}$ & Raiwand & Badian & Sharakpur & $\begin{array}{l}\text { Thokar } \\
\text { Niaz Baig }\end{array}$ & & & \\
\hline
\end{tabular}


Table 2 An inventory of floral diversity documented by local respondents ( $n=450$ ) from three districts (Faisalabad, Sargodha and Lahore) of Punjab, Pakistan for the treatment of equine disease

\begin{tabular}{|c|c|c|c|c|c|c|c|}
\hline $\begin{array}{l}\text { Sr. } \\
\text { No. }\end{array}$ & $\begin{array}{l}\text { Botanical name/ } \\
\text { English name }\end{array}$ & Local name & Family & Part (s) used & Usage diversity & $\begin{array}{l}\text { No. of } \\
\text { prescriptions }\end{array}$ & $\begin{array}{l}\text { Part of } \\
\text { remedies }\end{array}$ \\
\hline 1. & Acacia nilotica (L.) Willd. ex Delile & Desi kikar & Fabaceae & Branches & Internal parasites, swelling & 2 & 2 \\
\hline 2. & Allium cepa L. var. aggregatum G. Don & Piyaz & Liliaceae & Bulb & $\begin{array}{l}\text { Anorexia, bad habits, bronchitis, colic, diarrhea, fever, } \\
\text { heat stress, indigestion, pain, quidding, weakness }\end{array}$ & 11 & 33 \\
\hline 3. & Allium sativum L. & Lehsan & Liliaceae & Bulb & Bronchitis, fever, indigestion & 3 & 3 \\
\hline 4. & Aloe vera $(\mathrm{L}$.$) Burm. \mathrm{f}$. & $\begin{array}{l}\text { Kawar } \\
\text { gandal }\end{array}$ & Aloaceae & Leaves & Anorexia, bronchitis & 2 & 2 \\
\hline 5. & Amomum subulatum Roxb. & Ilaichi & Zingiberaceae & Fruit & Fever & 1 & 1 \\
\hline 6. & Anethum graveolens $\mathrm{L}$. & Soye & Umbelliferae & Seeds & $\begin{array}{l}\text { Anorexia, bronchitis, colic, fever, indigestion, } \\
\text { lameness, toxemia, weakness }\end{array}$ & 8 & 10 \\
\hline 7. & Azadirachta indica A. Juss. & Neem & Meliaceae & Leaves & Dermatitis, external parasites, lameness, wound & 5 & 13 \\
\hline 8. & Bambusa bambos L. & Bans & Bambusaceae & Leaves & Bronchitis & 1 & 1 \\
\hline 9. & $\begin{array}{l}\text { Brassica campestris L. ssp. napus Duthie and } \\
\text { Fuller }\end{array}$ & Surson & Brassicaseae & $\begin{array}{l}\text { Seeds, seed } \\
\text { oil }\end{array}$ & $\begin{array}{l}\text { Colic, dermatitis, diarrhea, external parasites, lameness, } \\
\text { retention of urine, swelling, weakness, wound }\end{array}$ & 8 & 47 \\
\hline 10. & Calotropis procera (Ait.) W.T.Ait. & Aak & Asclepiadaceae & Buds & Weakness & 1 & 1 \\
\hline 11. & Capsicum annum L. & Subz mirch & Solanaceae & Fruit & $\begin{array}{l}\text { Anorexia, bronchitis, fever, indigestion, lameness, quidding, } \\
\text { retention of urine, toxemia, weakness }\end{array}$ & 9 & 19 \\
\hline 12. & Cascuta reflexa Roxb. & Akas bail & Cuscutaceae & Whole plant & Lameness & 1 & 1 \\
\hline 13. & Cicer arietinum L. & $\begin{array}{l}\text { Kalay } \\
\text { chaney }\end{array}$ & Fabaceae & Seeds & Dermatitis, lameness, wound & 3 & 4 \\
\hline 14. & Citrullus colocynthis (L.) Schrad. & Kor tumma & Cucurbitaceae & Fruit & Anorexia, fever, indigestion, weakness & 4 & 9 \\
\hline 15. & Citrus limon (L.) Burm. & Nimbu & Rutaceae & Fruit & Lameness, wound & 2 & 2 \\
\hline 16. & Cocos nucifera $\mathrm{L}$. & Giri & Arecaceae & Oil & Lameness & 1 & 1 \\
\hline 17. & Curcuma longa $\mathrm{L}$. & Haldi & Zingiberaceae & Rhizome & External parasite, lameness, wound & 3 & 8 \\
\hline 18. & Eruca sativa Mill. & Tara mera & Cruciferae & $\begin{array}{l}\text { Seed, seed } \\
\text { oil }\end{array}$ & Dermatitis, external parasite, wound & 3 & 5 \\
\hline 19. & Eugenia caryophyllata Thunb. & Loung & Myrtaceae & Fruit & Anorexia, lameness, pain & 3 & 4 \\
\hline 20. & Euphorbia caducifolia Haines. & Danda thor & Euphorbiaceae & Branches & Colic & 1 & 1 \\
\hline 21. & Ficus religiosa $\mathrm{L}$. & Pipal & Moraceae & Bark & Swelling & 1 & 1 \\
\hline 22. & Foeniculum vulgare P. Mill. & Sounf & Apiaceae & Seeds & Indigestion, weakness & 2 & 2 \\
\hline 23. & Geranium wallichianum D. Don Ex Sweet & Ratan jot & Geraniaceae & Seeds & Lameness, wound & 2 & 4 \\
\hline 24. & Glycyrrhiza glabra L. & Mullathi & Fabaceae & Roots & Bronchitis & 1 & 7 \\
\hline 25. & Grewia asiatica L. & Falsa & Tiliaceae & Fruit & Lameness & 1 & 1 \\
\hline
\end{tabular}


Table 2 An inventory of floral diversity documented by local respondents ( $n=450)$ from three districts (Faisalabad, Sargodha and Lahore) of Punjab, Pakistan for the treatment of equine disease (Continued)

\begin{tabular}{|c|c|c|c|c|c|c|c|}
\hline 26. & Halorrhena pubescens Wall. ExG. Don. & Kuro & Apocynaceae & Bark & Heat stress & 1 & 1 \\
\hline 27. & Hordeum vulgare L. & Jow & Poaceae & Seeds & Heat stress & 1 & 1 \\
\hline 28. & Lagenaria siceraria Molina & Kuddo & Cucurbitaceae & Leaves & Internal parasite & 1 & 1 \\
\hline 29. & Lawsonia inermis $\mathrm{L}$. & Mehndi & Lythraceae & Leaves & Wound & 1 & 7 \\
\hline 30. & Lens culinaris Medik. & Masoor & Fabaceae & Seeds & Wound & 1 & 1 \\
\hline 31. & Lepidium sativum L. & Halion & Apiaceae & Seeds & Internal parasites, weakness & 2 & 2 \\
\hline 32. & Linum usitatissimum $\mathrm{L}$. & Alsi & Linaceae & Seeds & Internal parasites & 1 & 1 \\
\hline 33. & Mallotus philippinensis (Lamk.) Meull. Arg. & Kamela & Euphorbiaceae & Fruit & Anorexia, internal parasites & 2 & 4 \\
\hline 34. & Mangifera indica $\mathrm{L}$. & Aam & Anacardiaceae & Fruit & Lameness & 1 & 1 \\
\hline 35. & Medicago sativa $\mathrm{L}$. & Lusan & Papilionaceae & Leaves & Lameness & 1 & 1 \\
\hline 36. & Mentha longifolia (L.) Huds. & Podina & Lamiaceae & Leaves & Anorexia & 1 & 1 \\
\hline 37. & Myristica fragrans HOUTT. & Jaful & Myristicaceae & Fruit & Lameness, pain, tetanus & 3 & 5 \\
\hline 38. & Nicotiana tabacum L. & Tambaku & Solanaceae & Leaves & Colic, pain & 2 & 3 \\
\hline 39. & Nigella sativa $\mathrm{L}$. & Kalonji & Ranunculaceae & Seeds & Anorexia, bronchitis & 2 & 2 \\
\hline 40. & Olea europaea L. & Zaytoon & Oleaceae & Fruit & Lameness & 1 & 1 \\
\hline 41. & Oryza sativa L. & Chawal & Poaceae & Whole plant & Internal parasites & 1 & 1 \\
\hline 42. & Peganum harmala L. & Hurmil & Zygophyllaceae & Fruit & Anorexia, lameness, pain, swelling & 4 & 4 \\
\hline 43. & Pennisetum glaucum L. & Bajra & Poaceae & Whole & Lameness & 1 & 1 \\
\hline 44. & Picrorhiza kurroa Royle ex. Benth. & Kourdh & Scrofulariaceae & Rhizome & Anorexia, fever, indigestion, lameness, tetanus, weakness & 6 & 11 \\
\hline 45. & Piper betle $\mathrm{L}$. & Paan & Piperaceae & Leaves & Anorexia & 1 & 1 \\
\hline 46. & Piper nigrum L. & Kali mirch & Piperaceae & Pepper corn & Anorexia, bronchitis, toxemia & 3 & 5 \\
\hline 47. & Prunus dulcis Mill. & Badam & Rosaceae & Seed & Diarrhea & 1 & 1 \\
\hline 48. & Ricinus communis $\mathrm{L}$. & Arind & Euphorbiaceae & Flower oil & Internal parasites & 1 & 1 \\
\hline 49. & Rosa damascena Mill. & Gulab & Rosaceae & Flowers & Bronchitis & 1 & 4 \\
\hline 50. & Sesamum indicum $\mathrm{L}$. & Til & Pedaliaceae & $\begin{array}{l}\text { Seeds, seed } \\
\text { oil }\end{array}$ & Colic, dermatitis, lameness, pain & 4 & 7 \\
\hline 51. & Trachyspermum ammi (L.) Sprague ex Turrill. & Ajwain & Apiaceae & Seeds & $\begin{array}{l}\text { Anorexia, bronchitis, colic, fever, heat stress, } \\
\text { indigestion, lameness, retention of urine }\end{array}$ & 8 & 19 \\
\hline 52. & Trifolium alexandrinum $\mathrm{L}$. & Barseem & Papilionaceae & Whole plant & Wound & 1 & 1 \\
\hline 53. & Trigonella foenum-graecum $\mathrm{L}$. & Methray & Fabaceae & Seeds & Toxemia & 1 & 1 \\
\hline 54. & Triticum aestivum $\mathrm{L}$. & Gundum & Poaceae & Flour, grain & External parasites, lameness, weakness, wound & 4 & 5 \\
\hline
\end{tabular}


Table 2 An inventory of floral diversity documented by local respondents $(n=450)$ from three districts (Faisalabad, Sargodha and Lahore) of Punjab, Pakistan for the treatment of equine disease (Continued)

\begin{tabular}{|c|c|c|c|c|c|c|c|}
\hline 55. & Vernonia anthelmintica (L.) Willd. & Kali zeeri & Asteraceae & Seeds & $\begin{array}{l}\text { Anorexia, bronchitis, colic, diarrhea, heat stress, } \\
\text { indigestion, lameness, toxemia, + Weakness+ Wound }\end{array}$ & 10 & 23 \\
\hline 56. & Withania coagulans Dunal & Paneer & Solanaceae & Leaves & Anorexia, fever, indigestion, weakness & 4 & 4 \\
\hline 57. & Withania somnifera L. Dunal & Aksan & Solanaceae & Leaves & Wound & 1 & 1 \\
\hline 58. & Zea mays L. & Makai & Gramineae & Flour & Anorexia, haematuria, weakness, wound & 4 & 5 \\
\hline 59. & Zingiber officinale Roscoe & Adrak & Zingiberaceae & Rhizome & $\begin{array}{l}\text { Anorexia, bronchitis, colic, fever, heat stress, indigestion, } \\
\text { lameness, pain, tetanus, weakness }\end{array}$ & 10 & 26 \\
\hline 60. & Ziziphus jujuba L. Lam., non P. Mill. & Beri & Rhamnaceae & Leaves & Wound & 1 & 1 \\
\hline
\end{tabular}


Table 3 List of traditional veterinary practices based on plant materials for the treatment of different diseases/conditions of equines reported by the local respondents $(n=450)$ in the study area

S. Phytotherapeutic material used

Dose and administration

Usage

No.

Anorexia

1 Zingiber officinale (rhizome) + Capsicum annum (fruit) + Allium cepa (bulb) + Common salt

$50 g+125 g+250 g+125 g ;$ MGB/PO (Mix, Grind, and

Allium cepa (bulb) + Citrullus colocynthis (fruit) + Capsicum annum (fruit) + Zingiber officinale (rhizome) + Jaggery

3 Allium cepa (bulb) + Zingiber officinale (rhizome) + Black salt

4 Capsicum annum (fruit) + Allium cepa (bulb) + Vernonia anthelmintica (seeds) +

Eugenia caryophyllata (fruit) + Zingiber officinale (rhizome) + Common salt

Capsicum annum (fruit) + Allium cepa (bulb) + Zingiber officinale (rhizome) +

5 Trachyspermum ammi (seeds) + Nigella sativa (seeds) + Black salt + Common salt + Picrorhiza kurroa (rhizome) + Citrullus colocynthis (fruit) + Peganum harmala (fruit)

6 Common salt + Allium cepa (bulb) + Capsicum annum (fruit)

7 Common salt + Black salt + Allium cepa (bulb) + Piper nigrum (pepper corn) + Vernonia anthelmintica (seeds) + Trachyspermum ammi (seeds)

8 Zea mays (flour) + Brown sugar

9 Zea mays (flour) + Brown sugar + Water

10 Jaggery + Allium cepa (bulb)

11 Mallotus philippinensis (fruit) + Yogurt

12 Mentha longifolia (leaves) + Capsicum annum (fruit) + Common salt + Allium cepa (bulb)

13 Picrorhiza kurroa (rhizome) + Citrullus colocynthis (fruit) + Aloe vera (leaves) + Allium cepa (bulb)

14 Piper nigrum (pepper corn) + Black salt + Vernonia anthelmintica (seeds) + Capsicum annum (fruit)

15 Trachyspermum ammi (seeds) + Vernonia anthelmintica (seeds) + Jaggery

16 Trachyspermum ammi (seeds) + Zingiber officinale (rhizome) + Piper nigrum (pepper corn) +

Piper betle (leaves) + Jaggery

Vernonia anthelmintica (seeds) + Anethum graveolens (seeds) + Black salt + Zingiber officinale (rhizome) +

17 Withania coagulans (leaves) + Common salt + Picrorhiza kurroa (rhizome) + Citrullus colocynthis (fruit) + Jaggery + Allium cepa (bulb) + Capsicum annum (fruit)

18 Zingiber officinale (rhizome) + Allium cepa (bulb) + Capsicum annum (fruit) + make a Bolus to administer Per Oss)

$125 g+50 g+125 g+50 g+100 g ;$ MGB/PO

$250 \mathrm{~g}+100 \mathrm{~g}+100 \mathrm{~g} ; \mathrm{MGB} / \mathrm{PO}-2-3$ days

$500 g+250 g+250 g+100 g+250 g+250 g ; M G B / P O$

$125 g+125 g+50 g+25 g+50 g+50 g+50 g+50 g+$

$50 \mathrm{~g} ; \mathrm{MGB} / \mathrm{PO}$ - divide into 02 doses for 02 days

$125 \mathrm{~g}+250 \mathrm{~g}+125 \mathrm{~g} ; \mathrm{MGB} / \mathrm{PO}$

$50 g+50 g+50 g+25 g+25 g+25 g ; M G B / P O$

$250 \mathrm{~g}+250 \mathrm{~g} ; \mathrm{MGB} / \mathrm{PO}$

$250 \mathrm{~g}+250 \mathrm{~g}+5 \mathrm{~L} ;$ Mix and give PO

$250 \mathrm{~g}+500 \mathrm{~g} ; \mathrm{MGB} / \mathrm{PO}$

$10 \mathrm{~g}+125 \mathrm{~g} ; \mathrm{MGB} / \mathrm{PO}$

$125 g+50 g+50 g+125 g ; M G B / P O$

$125 \mathrm{~g}+125 \mathrm{~g}+50 \mathrm{~g} ; \mathrm{MGB} / \mathrm{PO}$

$50 \mathrm{~g}+25 \mathrm{~g}+25 \mathrm{~g}+25 \mathrm{~g} ; \mathrm{MGB} / \mathrm{PO}$

$25 g+50 g+125 g ; M G B / P O$

$50 g+50 g+50 g+25 g+250 g ;$ MGB/PO

$125 g+125 g+12 g+50 g+250 g+50 g+250 g+$

$500 g+250 g+250 g+250 g ; M G B / P O$

$125 \mathrm{~g}+1 \mathrm{~kg}+250 \mathrm{~g}+50 \mathrm{~g}+250 \mathrm{~g} ; \mathrm{MGB} / \mathrm{PO}$

\section{Total entries}

Bad habit (Mud eating)

1 Jaggery + Allium cepa (bulb)

$250 \mathrm{~g}+250$ g; Boiled bulbs mixed with jiggery - given PO

\section{Total entries}

\section{Bronchitis}

1 Glycyrrhiza glabra (roots) + Jaggery 
Table 3 List of traditional veterinary practices based on plant materials for the treatment of different diseases/conditions of equines reported by the local respondents $(n=450)$ in the study area (Continued)

\begin{tabular}{|c|c|c|c|}
\hline 2 & Jaggery + Allium cepa (bulb) & $250 \mathrm{~g}+250 \mathrm{~g} ; \mathrm{MGB} / \mathrm{PO}-2-3$ days & 4 \\
\hline 3 & Nuswar & $10 \mathrm{~g}$ nuswar pushed in nose with a pipe & 3 \\
\hline 4 & Glycyrrhiza glabra (roots) + Rosa damascena (flowers) + Piper nigrum (pepper corn) & $250 g+375 g+25 g ; M G B / P O-2-3$ days & 2 \\
\hline 5 & Glycyrrhriza glabra (roots) + Zingiber officinale (rhizome) + Allium cepa (bulb) + Jaggery & $50 g+50 g+125 g+125 g ; M G B / P O$ & 2 \\
\hline 7 & Bumbusa bambos (leaves) & $500 \mathrm{~g} \mathrm{PO}$ & 1 \\
\hline 8 & Glycyrrhiza glabra (roots) + Allium cepa (bulb) + Nigella sativa (seeds) + Ammonium chloride + Jaggery & $\begin{array}{l}250 \mathrm{~g}+2 \mathrm{~kg}+250 \mathrm{~g}+125 \mathrm{~g}+2 \mathrm{~kg} \text {; Mixed, ground and } \\
\text { fried to make custard }-125 \mathrm{~g} \text { daily for } 5 \text { days }\end{array}$ & 1 \\
\hline 9 & Glycyrrhriza glabra (roots) + Jaggery + Allium cepa (bulb) & $50 g+250 g+250 g ; M G B / P O$ & 1 \\
\hline 10 & $\begin{array}{l}\text { Glycyrrhiza glabra (roots) + Jaggery + Vernonia anthelmintica (seeds) + } \\
\text { Anethum graveolens (seeds) + Allium cepa (bulb) }\end{array}$ & $25 g+250 g+25 g+25 g+250 g ;$ MGB/PO & 1 \\
\hline 11 & Glycyrrhiza glabra (roots) + Rosa damascena (flowers) + Allium cepa (bulb) & $125 g+250 g+500 g ; M G B / P O$ & 1 \\
\hline 12 & $\begin{array}{l}\text { Glycyrrhiza glabra (roots) + Rosa damascena (flowers) + Ammonium chloride + } \\
\text { Piper nigrum (pepper corn) + Allium cepa (bulb) + Jaggery + Water }\end{array}$ & $\begin{array}{l}125 \mathrm{~g}+125 \mathrm{~g}+25 \mathrm{~g}+25 \mathrm{~g}+250 \mathrm{~g}+125 \mathrm{~g}+125 \mathrm{ml} \text {; Mixed, } \\
\text { fried and made custard }- \text { given PO }\end{array}$ & 1 \\
\hline 13 & Glycyrrhiza glabra (roots) + Rosa damascena (flowers) + Zingiber officinale (rhizome) & $125+125 \mathrm{~g}+125 \mathrm{~g} ; \mathrm{MGB} / \mathrm{PO}$ & 1 \\
\hline 14 & $\begin{array}{l}\text { Aloe vera (leaves) + Capsicum annum (fruit) + Zingiber officinale (rhizome) }+ \\
\text { Allium sativum (bulb) + Vernonia anthelmintica (seeds) }\end{array}$ & $125 g+125 g+50 g+50 g+50 g ;$ MGB/PO & 1 \\
\hline 15 & $\begin{array}{l}\text { Jaggery + Allium cepa (bulb) + Glycyrrhiza glabra (roots) + Zingiber officinale (rhizome) + } \\
\text { Piper nigrum (pepper corn) }\end{array}$ & $250 g+500 g+100 g+100 g+50 g ;$ MGB/PO & 1 \\
\hline 16 & Joshanda + Jaggery + Allium cepa (bulb) + Water & $1 \mathrm{~kg}+1 \mathrm{~kg}+2 \mathrm{~kg}+2 \mathrm{~L}$; Mixed, boiled in water till concentrated - given PO & 1 \\
\hline 17 & Rosa damascena (flowers) + Glycyrrhiza glabra (roots) + Ammonium chloride & $375 g+50 g+10 g ; M G B / P O$ & 1 \\
\hline 18 & Trachyspermum ammi (seeds) + Jaggery + Common salt & $100 \mathrm{~g}+200 \mathrm{~g}+50 \mathrm{~g} ; \mathrm{MGB} / \mathrm{PO}$ & 1 \\
\hline \multirow[t]{3}{*}{19} & Trachyspermum ammi (seeds) + Vernonia anthelmintica (seeds) + Jaggery & $25 g+25 g+125 g ; M G B / P O$ & 1 \\
\hline & Total entries & & 28 \\
\hline & Colic & & \\
\hline 1 & Brassica campestris (seed oil) & $500 \mathrm{ml}-\mathrm{PO}$ & 2 \\
\hline 2 & Brassica campestris (seed oil) + Water & $250 \mathrm{ml}+250 \mathrm{ml}-\mathrm{PO}$ & 2 \\
\hline 3 & Nicotiana tabacum (leaves) + Jaggery & $50 \mathrm{~g}+250 \mathrm{~g} ; \mathrm{MGB} / \mathrm{PO}$ & 2 \\
\hline 6 & Allium cepa (bulb) & 500 g juice $-P O$ & 1 \\
\hline 7 & Ammonium chloride + Brassica campestris (seed oil) + Common salt & $50 \mathrm{~g}+125 \mathrm{ml}+50 \mathrm{~g} ;$ Mix and give PO & 1 \\
\hline 8 & Ammonium chloride + Potassium bicarbonate & $50 \mathrm{~g}+50 \mathrm{~g} ; \mathrm{MGB} / \mathrm{PO}$ & 1 \\
\hline 9 & Euphorbia caducifolia (branches) + Water & $10 \mathrm{ml}$ juice mixed in $250 \mathrm{ml}$ water - PO & 1 \\
\hline 11 & Jaggery + Allium cepa (bulb) & $250 \mathrm{~g}+250 \mathrm{~g} ; \mathrm{MGB} / \mathrm{PO}$ & 1 \\
\hline 12 & Jaggery + Nicotiana tabacum (leaves) & $250 \mathrm{~g}+50 \mathrm{~g} ;$ Mix and give PO & 1 \\
\hline 13 & Sodium carbonate + Jaggery +7 up & $125 \mathrm{~g}+250 \mathrm{~g}+250 \mathrm{ml}$; Bolus followed by 7up & 1 \\
\hline
\end{tabular}


Table 3 List of traditional veterinary practices based on plant materials for the treatment of different diseases/conditions of equines reported by the local respondents $(n=450)$ in the study area (Continued)

\footnotetext{
14 Potassium nitrate + Potassium bicarbonate + Hukka water

15 Trachyspermum ammi (seeds) + Soap + Common salt + Water

16 Sesamum indicum (seeds)

17 Trachyspermum ammi (seeds) + Anethum graveolens (seeds) + Jaggery + White salt

Trachyspermum ammi (seeds) + Vernonia anthelmintica (seeds) + Jaggery + Brassica campestris (seed oil) + Water

19 Vernonia anthelmintica (seeds) + Zingiber officinale (rhizome) + Milk + Water
}

\section{Total entries}

\section{Dermatitis}

1 Brassica campestris (seed oil)

2 Hukka water

3 Eruca sativa (seed oil)

4 Eruca sativa (seed oil) + Sulfur

6 Cicer arietinum (seeds) + Eruca sativa (seed oil)

7 Azadirachta indica (leaves) + Alum + Common salt + Water

8 Brassica campestris (seed oil) + Eruca sativa (seed oil)

9 Brassica campestris (seed oil) + Jaggery

10 Brassica campestris (seed oil) + Yogurt

13 Eruca sativa (seeds) + Common salt

14 Sesamum indicum (seed oil)

\section{Total entries}

\section{Diarrhea}

1 Allium cepa (bulb) + Common salt

2 Citrullus colocynthus (fruit) + Vernonia anthelmintica (seeds) + Black salt + Common salt

3 Milk + Brassica campestris (seed oil)

4 Prunus dulcis (seeds) + Jaggery

5 Vernonia anthelmintica (seeds) + Black salt + Citrillus colocynthus (fruit)

\section{Total entries}

\section{External parasite}

3 Azadirachta indica (leaves) + Water

4 Brassica campestris (seed oil) + Sump oil

5 Brassica campestris (seed oil) + Kerosene oil

6 Curcuma longa (rhizome)
$25 \mathrm{~g}+25 \mathrm{~g}+250 \mathrm{ml}$; Mix and give PO

$125 g+125 g+250 g+2 \mathrm{~L}$; Decoction given PO

$500 \mathrm{ml} \mathrm{PO}$

$50 g+50 g+250 g+125 g ; M G B / P O$

$50 \mathrm{~g}+50 \mathrm{~g}+250 \mathrm{~g}+250 \mathrm{ml}+250 \mathrm{ml}$; Decoction PO

$50 \mathrm{~g}+50 \mathrm{~g}+250 \mathrm{ml}+250 \mathrm{ml}$; Grind and give PO

50-100 ml; Topical application

Topical application

$100 \mathrm{ml}$; Topical application

$250 \mathrm{ml}+50 \mathrm{~g}$; Topical application

$250 \mathrm{~g}+250 \mathrm{ml} ; \mathrm{GMB} / \mathrm{PO}$

$250 g+25 g+50 g+3$ L; Decoction applied topically

$50 \mathrm{ml}+50 \mathrm{ml}$; Topical application (massage)

$50 \mathrm{ml}+50 \mathrm{~g}$; Topical application

Topical application

Topical application

$50 \mathrm{ml}$; Topical application

2 bulbs +50 g; MGB/PO

$50 g+50 g+50 g+50 g ; M G B / P O$

$250 \mathrm{ml}+125 \mathrm{ml}$; Decoction given PO

7-10 seeds + 250 g; MGB/PO

$50 g+50 g+50 g ; M G B / P O$

$250 \mathrm{~g}+1 \mathrm{~L}$; Topical application of decoction

$125 \mathrm{ml}+125 \mathrm{ml}$; Topical application

$250 \mathrm{ml}+125 \mathrm{ml}$; Topical application

Topical application of powder 
Table 3 List of traditional veterinary practices based on plant materials for the treatment of different diseases/conditions of equines reported by the local respondents $(n=450)$ in the study area (Continued)

\begin{tabular}{|c|c|c|}
\hline 7 & Eruca sativa (seeds) + Triticum aestivum (flour) & $50 \mathrm{~g}+250 \mathrm{~g} ; \mathrm{MGB} / \mathrm{PO}$ \\
\hline \multirow[t]{5}{*}{9} & Brassica campestris (seeds) & Topical application of ground seeds \\
\hline & Total entries & \\
\hline & Eye problem & \\
\hline & Total entries & \\
\hline & Fever & \\
\hline 1 & $\begin{array}{l}\text { Vernonia anthelmintica (seeds) + Anethum graveolens (seeds) + Black salt + Zingiber officinale (rhizome) + } \\
\text { Withania coagulans (leaves) + Common salt + Picrorhiza kurroa (rhizome) + Citrullus colocynthis (fruit) + } \\
\text { Jaggery + Allium cepa (bulb) + Capsicum annum (fruit) }\end{array}$ & $\begin{array}{l}125 g+125 g+12 g+50 g+250 g+50 g+250 g+500 g+ \\
250 g+250 g+250 g ; G M B \text { for } 8 \text { days and give in equal doses PO }\end{array}$ \\
\hline 2 & $\begin{array}{l}\text { Allium cepa (bulb) + Capsicum annum (fruit) + Zingiber officinale (rhizome) + Jaggery + Allium sativum } \\
\text { (bulb) }\end{array}$ & $125 g+50 g+25 g+125 g+25 g ; G M B / P O$ \\
\hline 3 & Black salt + Trachyspermum ammi (seeds) & $125 g+50 g P O$ \\
\hline 4 & Common salt + Trachyspermum ammi (seeds) + Water & $50 \mathrm{~g}+50 \mathrm{~g}+50 \mathrm{ml} ; \mathrm{MGB} / \mathrm{PO}$ \\
\hline 7 & Jaggery + Amomum subulatum (fruit) & $250 \mathrm{~g}+50 \mathrm{~g} ; \mathrm{MGB} / \mathrm{PO}$ \\
\hline \multirow[t]{2}{*}{9} & Piper nigrum (pepper corn) + Trachyspermum ammi (seeds) + Water + Sugar & $\begin{array}{l}500 \mathrm{~g}+100 \mathrm{~g}+500 \mathrm{ml}+1 \mathrm{~kg} ; \text { Mix, fry and make custard }- \\
250 \mathrm{~g} \text { daily for } 5 \text { days }\end{array}$ \\
\hline & Haematuria & \\
\hline 1 & Butter + Piper nigrum (pepper corn) & $125 \mathrm{~g}+25 \mathrm{~g} ; \mathrm{MGB} / \mathrm{PO}$ \\
\hline 2 & Zea mays (flour) + Brown sugar + Water & $500 g+500 g+2 L P O$ \\
\hline \multirow[t]{3}{*}{3} & Potassium bicarbonate + Potassium nitrate & $50 \mathrm{~g}+50 \mathrm{gPO}$ \\
\hline & Total entries & \\
\hline & Heat stress & \\
\hline 1 & Trachyspermum ammi (seeds) + Common salt & $50 \mathrm{~g}+125 \mathrm{~g}$; Soak seeds overnight, grind and mix with salt - give PO \\
\hline 2 & Allium cepa (bulb) + Jaggery & $250 \mathrm{~g}+250 \mathrm{~g}$; Half boil bulbs, mix in jiggery and give PO \\
\hline 3 & Dalda ghee + Vernonia anthelmintica (seeds) & $125 \mathrm{~g}+50 \mathrm{~g} ; \mathrm{MGB} / \mathrm{PO}$ \\
\hline 4 & Trachyspermum ammi (seeds) + Jaggery & $250 \mathrm{~g}+250 \mathrm{~g} P O$ \\
\hline 5 & Vernonia anthelmintica (seeds) + Sugar & $50 \mathrm{~g}+125 \mathrm{~g} ; \mathrm{MGB} / \mathrm{PO}$ \\
\hline 6 & Water & As much as animal can drink \\
\hline 7 & Zingiber officinale (rhizome) + Halorrhena pubescens $($ bark $)+$ Black salt + Common salt + Water & $50 \mathrm{~g}+50 \mathrm{~g}+25 \mathrm{~g}+25 \mathrm{~g}+1 \mathrm{~L}$; Soak all in earthen utensil for $24 \mathrm{hrs}$; PO \\
\hline 8 & Hordeum vulgare (seeds) + Water & $2 \mathrm{~kg}+4 \mathrm{~L} ;$ Soak overnight; PO \\
\hline
\end{tabular}

1 Butter + Piper nigrum (pepper corn)

2 Zea mays (flour) + Brown sugar + Water

$500 g+500 g+2 L P O$
$50 g+50 g P O$ 
Table 3 List of traditional veterinary practices based on plant materials for the treatment of different diseases/conditions of equines reported by the local respondents $(n=450)$ in the study area (Continued)

\section{Total entries}

Indigestion

1. Capsicum annum (fruit) + Allium cepa (bulb) + Vernonia anthelmintica (seeds) + Trachyspermum ammi (seeds) + Zingiber officinale (rhizome) + Jaggery + Citrullus colocynthis (fruit) + Black salt + Common salt

Citrullus colocynthis (fruit) + Picrorhiza kurroa (rhizome) + Vernonia anthelmintica (seeds) +

Black salt + Ammonium chloride

3 Jaggery + Trachyspermum ammi (seeds) + Zingiber officinale (rhizome) + Allium cepa (bulb) + Allium sativum (bulb)

Vernonia anthelmintica (seeds) + Anethum graveolens (seeds) + Black salt + Zingiber officinale (rhizome) +

4 Withania coagulans (leaves) + Common salt + Picrorhiza kurroa (rhizome) + Citrullus colocynthis (fruit) + Jaggery + Allium cepa (bulb) + Capsicum annum (fruit)

5 Zingiber officinale (rhizome) + Trachyspermum ammi (seeds) + Foeniculum vulgare (seeds) + Black salt + Jaggery

\section{Total entries}

Internal parasites

1 Mollotus philipinensis (fruit) + Jaggery

2 Mallotus philippinensis (fruit) + Yogurt

3 Acacia nilotica (branches) + Jaggery

4 Jaggery + Azadirachta indica (leaves)

5 Jaggery + Oryza sativa (whole plant)

6 Jaggery + Yogurt + Mollotus philpinensis (fruit)

7 Lagenaria siceraria (leaves)

8 Linum usitatissimum (seeds) + Lepidium sativum (seeds) + Jaggery + Mallotus philippinensis (fruit)

9 Mallotus philippinensis (fruit) + Milk whey

10 Nurru (stem) + Jaggery

11 Ricinus cummunis (flower oil)

12 Ricinus cummunis (flower oil)

\section{Total entries}

\section{Lameness}

1 Brassica campestris (seed oil)

2 Sesamum indicum (seed oil)

3 Copper sulfate + Jaggery

5 Citrus limon (fruit)

7 Brassica campestris (seed oil) + Kerosene oil

12 Azadirachta indica (leaves) + Common salt + Water

$50 g+250 g+50 g+50 g+50 g+100 g+$

$100 \mathrm{~g}+100 \mathrm{~g}+100 \mathrm{~g} ; \mathrm{MGB} / \mathrm{PO}$

$100 g+10 g+10 g+50 g+3$ balls; Mixed in hot water and given PO 1

$250 g+50 g+50 g+250 g+50 g ; P O$

$125 g+125 g+12 g+50 g+250 g+50 g+250 g+500 g+250 g+1$

$250 \mathrm{~g}+250 \mathrm{~g} ; \mathrm{MGB} / \mathrm{PO}$, equally divided in 8 balls and given one daily

$50 g+50 g+50 g+50 g+250 g ; M G B / P O$

$50 \mathrm{~g}+250 \mathrm{gPO}$

$10 \mathrm{~g}+125 \mathrm{gPO}$

$125 g+125 g P O$

$250 \mathrm{~g}+500 \mathrm{~g}$; Jaggery 10 minutes before $A$. indica leaves PO

$250 \mathrm{~g}+1 \mathrm{~kg}$; Give Jaggery on first day and $O$. sativa next day

$125 g+250 g+50 g$ PO

$250 \mathrm{~g}$; Ground and given PO

$60 g+60 g+250 g+25 g ; P O$

$10 \mathrm{~g}+250 \mathrm{ml} \mathrm{PO}$

$250 \mathrm{~g}+250 \mathrm{~g}$; Given jaggery first and then nurru

$250 \mathrm{ml}$ oil PO

$250 \mathrm{ml} \mathrm{PO}$

50-100 ml; Topical application 
Table 3 List of traditional veterinary practices based on plant materials for the treatment of different diseases/conditions of equines reported by the local respondents $(n=450)$ in the study area (Continued)

\footnotetext{
13 Azadirachta indica (leaves) + Water

14 Azadirachta indica (leaves) + Water + Common salt + Alum

15 Brassica campestris (seed oil) + Kerosene oil + Capsicum annum (fruit)

16 Brassica campestris (seed oil)

17 Cascuta reflexa (whole plant) + Ghee

18 Cicer arietinum (seeds)

19 Cicer arietinum (seeds) + Jute bag

21 Common salt + Triticum aestivum (flour) + Water

22 Geranium wallichianum (seeds) + Brassica campestris (seed oil)

23 Geranium wallichianum (seeds) + Brassica campestris (seed oil)

24 Jaggery + Curcuma longa (rhizome)

27 Medicago sativa (leaves) + Common salt

28 Mangifera indica (fruit)

29 Myristica fragrans (fruit) + Geranium wallichianum (seeds) + Eugenia caryophyllata (fruit) + Sesamum indicum (seeds oil) + Brassica campestris (seed oil)

30 Olea europaea (fruit) + Cocus nucifera (oil) + Fish + Sump oil

31 Pennisetum glaucum (whole) + Jaggery

32 Peganum harmala (fruit) + Alum + Jaggery

34 Sesamum indicum (seeds) + Eugenia caryophyllata (fruit) + Myristica fragrans (fruit)

35 Trachyspermum ammi (seeds) + Anethum graveolens (seeds) + Vernonia anthelmintica (seeds) + Jaggery

36 Vernonia anthelmintica (seeds) + Anethum graveolens (seeds)

37 Water + Triticum aestivum (flour) + Common salt + Alum

38 Zingiber officinale (rhizome) + Picrorhiza kurroa (rhizome) + Vernonia anthelmintica (seeds) + Grewia asiatica (fruit) + Jaggery

39 Ricinus cummunis (bark) + Sesamum indicum (seeds oil)
}

\section{Total entries}

\section{Pain associated with infection}

1 Sesamum indicum (seed oil) + Eugenia caryophyllata (fruit) + Myristica fragrans (fruit)

2 Sodium carbonate + Jaggery

3 Nicotiana tabacum (leaves) + Jaggery

Zingiber officinale (rhizome) + Allium cepa (bulb) + Glycyrrhiza glabra (roots) + Capsicum annum (fruit) + Water

5 Alum + Peganum harmala (fruit) + Jaggery
$100 g+1$ L-Topical application of decoction

$250 \mathrm{~g}+4 \mathrm{~L}+1 \mathrm{~kg}+250 \mathrm{~g}$; Topical application of decoction

$50 \mathrm{ml}+50 \mathrm{ml}+25 \mathrm{~g}$; Topical application

Topical application of semi-hot oi

C. reflexa fried in oil; Topical application

$50 \mathrm{~g}$ grains tied on affected area by putting in a cloth bag

$250 \mathrm{~g}$ grains tied on affected area by putting in a jute bag

Topical application of hot mixture

$50 \mathrm{~g}+50 \mathrm{ml} ;$ Mix, fry and apply topically

$50 \mathrm{~g}+50 \mathrm{ml}$; Mix, fry and apply topically

$250 \mathrm{~g}+50 \mathrm{~g} ; \mathrm{MGB} / \mathrm{PO}$

$500 \mathrm{~g}+100 \mathrm{~g} ;$ Mix and apply on the affected area for 2-3 days Topical application on the lesion

$50 \mathrm{~g}+50 \mathrm{~g}+50 \mathrm{~g}+250 \mathrm{ml}+250 \mathrm{ml}$; Topical application

$250 \mathrm{ml}+250 \mathrm{ml}+250 \mathrm{ml}+250 \mathrm{ml}$; Topical application

$125 \mathrm{~g}+125 \mathrm{~g} ; \mathrm{MGB} / \mathrm{PO}$

Topical application

$125 \mathrm{ml}+10 \mathrm{~g}+50 \mathrm{~g}$; Topical hot application

$125 g+125 g+50 g+500 g ; M G B / P O$

$250 \mathrm{~g}+250 \mathrm{~g} ; 50 \mathrm{~g}$ daily

$2 \mathrm{~L}+500 \mathrm{~g}+125 \mathrm{~g}+25 \mathrm{~g}$; Topical application of decoction

$250 g+250 g+250 g+250 g+250 g ;$ GMB/PO

$250 \mathrm{~g}+250 \mathrm{ml}$; Boiled bark in seed oil and applied on affected part for 2-3 days

$125 \mathrm{ml}+25 \mathrm{~g}+25 \mathrm{~g}$; Mixed, fried and applied topically

One spoon + $250 \mathrm{~g} ; \mathrm{MGB} / \mathrm{PO}$

$50 \mathrm{~g}+250 \mathrm{~g} ; \mathrm{MGB} / \mathrm{PO}$

$1 \mathrm{~kg}+3 \mathrm{~kg}+1 \mathrm{~kg}+1 \mathrm{~kg}+1$ litre; MGB/PO

$50 g+125 g+125$ g; Mix, fry and give PO one spoon a day 
Table 3 List of traditional veterinary practices based on plant materials for the treatment of different diseases/conditions of equines reported by the local respondents $(n=450)$ in the study area (Continued)

\section{Total entries \\ Quidding}

1 Capsicum annum (fruit) + Jaggery + Common salt + Allium cepa (bulb)

\section{Total entries}

Retention of urine

1 Capsicum annum (fruit)

2 Brassica campestris (seed oil) + Milk

3 Brassica campestris (seed oil) + Water

7 Trachyspermum ammi (seeds)

\section{Total entries}

\section{Swelling}

1 Acacia nilotica (branches) + Ficus religiosa (bark) + Water + Brassica campestris (seed oil)

2 Azadirachta indica (leaves) + Alum + Common salt + Water

3 Leather + Brassica campestris (seed oil)

4 Peganum harmala (fruit)

Total entries

\section{Tetanus}

1 Egg + Zingiber officinale (rhizome) + Myristica fragrans (fruit) + Picrorhiza kurroa (rhizome)

2 Myristica fragrans (fruit) + Picrorhiza kurroa (rhizome) + Jaggery

\section{Total entries}

\section{Toxemia}

1 Capsicum annum (fruit) + Common salt

2 Capsicum annum (fruit) + Ghee + Water

3 Capsicum annum (fruit) + Ghee

4 Capsicum anuum (fruit) + Trigonella foenum-graecum (seeds) +

Vernonia anthelmintica (seeds) + Anethum graveolens (seeds) + Water

5 Piper nigrum (pepper corn) + Ghee

6ernonia anthelmintica (seeds) + Anethum graveolens (seeds) + Trigonella foenum-graecum (seeds) + Capsicum anuum (fruit) + Water

\section{Total entries}

\section{Weakness}

Vernonia anthelmintica (seeds) + Anethum graveolens (seeds) + Black salt + Zingiber officinale (rhizome) + Withania coagulans (leaves) + Common salt + Picrorhiza kurroa (rhizome) + Citrullus colocynthis (fruit) + Jaggery + Allium cepa (bulb) + Capsicum annum (fruit)
$50 g+250 g+50 g+250 g ; M G B / P O$

Applied Capsicum annum (fruit) L. on urethral opening

$125 \mathrm{ml}+250 \mathrm{ml} ; \mathrm{PO}$

$250 \mathrm{ml}+250 \mathrm{ml}$; given decoction PO

$125 \mathrm{~g}$ seeds soaked overnight in water and given $\mathrm{PO}$

$250 \mathrm{~g}+250 \mathrm{~g}+2 \mathrm{~L}+25 \mathrm{ml}$; Topical application of decoction

$250 \mathrm{~g}+50 \mathrm{~g}+1$ spoon $+2 \mathrm{~L}$; Topical application of decoction

$500 \mathrm{~g}+500 \mathrm{ml}$; Heated leather in seed oil and applied on inflammation

$125 \mathrm{~g} \mathrm{PO}$

One $+25 g+$ One seed $+25 g ; M G B / P O-3$ days

One fruit $+25 g+10$; MGB/PO and cuts on nose for bleeding

$250 \mathrm{~g}+125 \mathrm{~g} ; \mathrm{PO}$

$250 \mathrm{~g}+250 \mathrm{~g}+1000 \mathrm{ml}$; decoction $\mathrm{PO}$

$250 \mathrm{~g}+250 \mathrm{~g} ;$ Ground, fried and given PO

$1000 \mathrm{~g}+500 \mathrm{~g}+250 \mathrm{~g}+250 \mathrm{~g}+10$ litre; Boiled and made custard $200 \mathrm{~g}$ daily

$125 \mathrm{~g}+125 \mathrm{~g}$; Ground, mixed in warm ghee and given PO 1

$250 g+500 g+500 g+500 g+5$ litre; Decoction PO 
Table 3 List of traditional veterinary practices based on plant materials for the treatment of different diseases/conditions of equines reported by the local respondents $(n=450)$ in the study area (Continued)

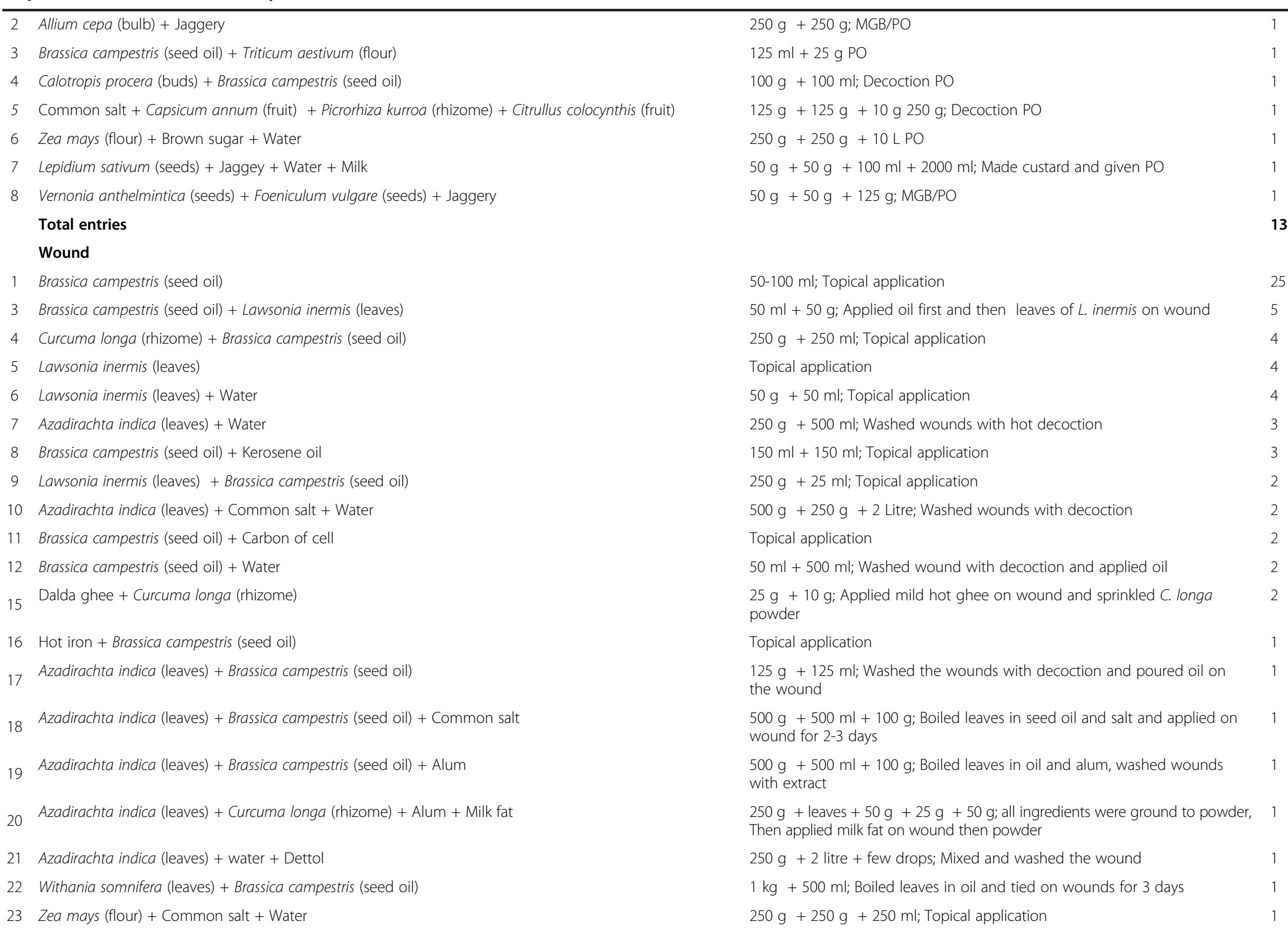


Table 3 List of traditional veterinary practices based on plant materials for the treatment of different diseases/conditions of equines reported by the local respondents $(n=450)$ in the study area (Continued)

\begin{tabular}{|c|c|c|c|}
\hline 24 & Lens culinaris (seeds) + Brassica campestris (seed oil) & $250 \mathrm{~g}+250 \mathrm{ml}$; Topical application of decoction & 1 \\
\hline 25 & Brassica campestris (seed oil) + Kerosene oil + Curcuma longa (rhizome) & $50 \mathrm{ml}+50 \mathrm{ml}+100 \mathrm{~g} ;$ Topical application & 1 \\
\hline 26 & Brassica campestris (seed oil) + Soap & $125 \mathrm{ml}$; Washed wound with soap and applied oil & 1 \\
\hline 27 & Trifolium alexandrinum (whole) & $\begin{array}{l}\text { Put Trifolium alexandrinum in closed utensils for hours and after that tied } \\
\text { on wound }\end{array}$ & 2 \\
\hline 28 & Capsicum anuum (fruit) & $50 \mathrm{~g}$; Topical application of powder & 1 \\
\hline 29 & Cicer arietinum (seeds) & $50 \mathrm{~g}$; wraped cloth and tied on wound, kept on pouring water on cloth & 1 \\
\hline 33 & Citrus limon (fruit) & Cut into half and rubbed on wound & 1 \\
\hline 31 & Curcuma longa (rhizome) & $10 \mathrm{~g}$; Topical application & 1 \\
\hline 32 & Eruca sativa (seeds) + Brassica campestris (seeds) & Topical application & 1 \\
\hline 33 & Geranium wallichianum (seeds) + Brassica campestris (seed oil) & $50 \mathrm{~g}+250 \mathrm{ml}$; Topical application & 1 \\
\hline 34 & Ghee + Curcuma longa (rhizome) & $25 \mathrm{~g}+25 \mathrm{~g}$; Mixed, fried and applied topically & 1 \\
\hline 35 & Lawsonia inermis (leaves) + Alum & $50 \mathrm{~g}+50 \mathrm{~g}$; Topical application & 1 \\
\hline 36 & Lawsonia inermis (leaves) + Alum + Brassica campestris (seed oil) & $125 \mathrm{~g}+50 \mathrm{~g}+50 \mathrm{ml}$; Topical application & 1 \\
\hline 37 & Lawsonia inermis (leaves) + Alum + Water & $\begin{array}{l}250 \mathrm{~g}+50 \mathrm{~g}+200 \mathrm{ml} \text {; Heated the alum, ground and mixed with water } \\
\text { and L. inermis; Topical application }\end{array}$ & 1 \\
\hline 38 & Leather + Brassica campestris (seed oil) & Burnt leather to ash, mixed with brassica oil and applied on wound & 1 \\
\hline 39 & Vernonia anthelmintica (seeds) + Triticum aestivum (flour) & $125 g+125 g P O$ & 1 \\
\hline 40 & Ziziphus jujube (leaves) + Brassica campestris (seed oil) & $250 \mathrm{~g}+250 \mathrm{ml}$; Boiled leaves in seed oil and tied on wounds for 2-3 days & 1 \\
\hline
\end{tabular}


Table 4 Frequency of plants used for the treatment of equine diseases/ conditions documented by local veterinary healers from three districts (Faisalabad, Sargodha and Lahore) of Punjab, Pakistan

\begin{tabular}{|c|c|c|c|c|}
\hline Conditions & Plants & Entries & $\begin{array}{l}\text { Prescriptions/ } \\
\text { remedies }\end{array}$ & Plants as part of prescriptions for the same disease more than one time ${ }^{[1]}$ \\
\hline Anorexia & 17 & 23 & 19 & $\begin{array}{l}\text { Allium (A.) cepa (12), Capsicum (Cp.) annum (9), Zingiber (Z.) officinale (8), Vernonia (V.) anthelmintica (6), Citrullus (Ct.) colocynthis (4), } \\
\text { Trachyspermum (T.) ammi (4), Picrorhiza (Pr.) kurroa (3), Piper (P.) nigrum (3), Zea mays (2) }\end{array}$ \\
\hline Bad habits & 1 & 5 & 2 & - \\
\hline Bronchitis & 12 & 33 & 21 & Glycyrrhiza glabra (11), A. cepa (8), Rosa damascena (5), Z. officinale (4), P. nigrum (2), T. ammi (2), V. anthelmintica (2) \\
\hline Colic & 9 & 24 & 21 & Brassica (B.) campestris (4), T. ammi (3), A. cepa (2), Nicotiana tabacum (2), V. anthelmintica (2) \\
\hline Dermatitis & 6 & 32 & 16 & Eruca sativa (5), B. campestris (4) \\
\hline Diarrhoea & 5 & 5 & 5 & Ct. colocynthus (2), V. anthenthelmintica (2) \\
\hline Ectoparasites & 5 & 23 & 11 & B. campestris (2) \\
\hline Eye problem & - & 2 & 2 & - \\
\hline Fever & 11 & 12 & 9 & T. ammi (3), A. cepa (2), Capsicum (Cp)annum (2), Z. officinale (2) \\
\hline Haematuria & 2 & 4 & 4 & - \\
\hline Heat stress & 6 & 10 & 8 & T. ammi (2), V. anthelmintica (2) \\
\hline Indigestion & 11 & 6 & 6 & Z. officinale (4), A. cepa (3), T. ammi (3), Cp. annum (2), Ct. colocynthis (2), Pr. kurroa (2), V. anthelmintica (2) \\
\hline Endoparasites & 8 & 31 & 12 & Molottus philpinensis (4), Ricinus cummunis (2) \\
\hline Lameness & 21 & 58 & 40 & $\begin{array}{l}\text { B. campestris (7), Sesamum indicum (4), Azadirachta (Az.) indica (3), Geranium wallichianum (3), V. anthelmintica (3), Anethum (An.) graveolens (2), } \\
\text { Cicer arietinum (2), Eugenia caryophllata (2), Myristica (M.) fragrans (2) }\end{array}$ \\
\hline Pain & 9 & 5 & 5 & - \\
\hline Quidding & 2 & 3 & 3 & - \\
\hline Urine retention & 3 & 10 & 8 & B. campestris (2) \\
\hline Swelling & 5 & 11 & 7 & B. campestris (2) \\
\hline Tetanus & 3 & 2 & 2 & M. fragrans (2), Pr. kurroa (2) \\
\hline Toxemeia & 5 & 7 & 7 & Cp. annum (5), An. graveolens (2), Trigonella foenum-graceum (2), V. anthelmintica (2) \\
\hline Weakness & 14 & 23 & 13 & A. cepa (2), B. campestris (2), Cp. annum (2), Ct. colocynthis (2), Pr. kurroa (2), V. anthelmintica (2) \\
\hline Wound & 16 & 121 & 57 & B. campestris (20), Az. indica (7), Lawsonia inermis (6), Curcuma longa (5) \\
\hline
\end{tabular}

${ }^{[1]}$ Plants were used in more than one prescription because of difference either in their dose, composition of the prescription, mode of preparation and administration, vehicles, etc. 
$(\mathrm{n}=11)$, fever $(\mathrm{n}=09)$, heat stress and retention of urine $(\mathrm{n}=08)$, swelling and toxemia $(\mathrm{n}=07)$, indigestion $(\mathrm{n}=06)$, diarrhoea and pain $(n=05)$, haematuria $(n=04)$, quidding $(\mathrm{n}=03)$, bad habits, eye problem and tetanus $(\mathrm{n}=02)$. Similar trend was seen for the number of TVPs used for different diseases/conditions being highest $(n=121)$ for wounds and the lowest for tetanus $(n=02)$. Maximum number of plants were used against lameness $(n=21)$ followed by anorexia $(n=17)$, wounds $(n=16)$, weakness $(n=14)$, bronchitis $(n=12)$, etc. as shown in the Table 4 .

\section{Approaches adapted in plant usage}

Seeds were the most frequently used $(n=16 / 60)$ part of plants as such or as their oils followed by leaves $(n=12$ / $60)$ and fruit $(n=11 / 60)$. The other parts of plants used were: whole plant, rhizome, bark, branches, bulb, buds, flour, pepper corn, roots, etc (Table 2). Prescriptions for treatment/control of different conditions of equines were based on single or multiple plants. Most of the recipes were prepared by mixing and grinding the ingredients. The powder was then made into physic balls as bolus to be given orally or decoctions were prepared for drenching the animals and/or used for topical application as washing, spraying, ointment, liniment, massage, etc. The prescriptions also differed in dose, method of preparation, and mode of administration of plants and/or materials other than plants; within and amongst the diseases/conditions. In some cases, frying, burning (to create smoke around animals), pouring, drinking, soaking before use, forced inhalation and steaming of ingredients were practiced.

\section{Discussion}

There are several recent evidences of plant based treatment and control strategies from Pakistan, especially for parasitism $[11,12]$. This has been supported by repellent activity of Moringa oleifera [17], an indication to be used against ecto-parasites. Plants from different geographical regions have produced variable results [18] as the synthesis of secondary plant products can be affected by environmental/growing conditions. Steroid saponins show pharmacologic actions like antifungal, antibacterial, anti-inflammatory and hypocholesteremic influences (Wang et al., [19]). So, plants producing saponins and organosulfur compounds like those of genus Allium can be used in the conditions described above.

Large number of qualified veterinarians also advocates the use of phytotherapy, other than preventive medication, but these practices are less organized in the form of scientific reports and are usually transferred orally as these have been developed by farmers, rather than by scientists in sophisticated laboratories. This situation is typical of a rural underdeveloped culture like that of pastoralists of Africa [20] and other parts of world having dependence on phytotherapy for their animals. Inadequate access to modern health care facilities due to cost-ineffectiveness, inherited beliefs, empirical evidence of efficacy, cultural acceptability and availability of botanicals at the farmers' doorstep are the main factors [21] that lead to dependence of livestock farmers on the phytotherapy. The indigenous knowledge and skills can contribute towards development of phytotherapy in less developed areas of the world [22,23]. Plants are considered to possess relatively higher bioactive secondary compounds, thus hold promise for drug discovery. Most of the plant-derived chemicals are secondary metabolites, of which at least 12,000 have been isolated; a number estimated to be less than $10 \%$ of the total [24]. Nok et al. [25,26] and Nok and Williams [27] have discussed the active principles as well as the mechanisms of action of some plant extracts that are used in phytotherapy.

Fruitful efforts have also been made previously to document TVPs in some parts of Punjab, Pakistan focusing livestock but not including equines [28-32]. Equine industry has the ready and largely uninformed access to herbal products. Therefore, at least to the extent of equines, herbals are more than traditional veterinary medicine. The researchers have, therefore, focused on documentation and validation of usage of plants based on the claims of traditional healers [33-37].

Results of the present study have revealed that equine owners and/or traditional veterinary healers have great wealth of indigenous knowledge based on their practices and experience, which is evident from the number of plants $(n=60)$ used for treatment of different diseases/ conditions of equines in Faisalabad, Sargodha and Lahore. Use of plants in multiple disorders indicates diversity of their pharmacological and toxicological impacts [38]. Plants have more than one mode of actions; therefore, provide broad spectrum activities in different diseases [39] due to diversity of phyto - chemicals. Twenty - four plants including Anethum graveolens, Bambusa bambos, Cascuta reflexa, Citrus limon, Cocus nucifera, Ficus religiosa, Geranium wallichianum, Grewia asiatica, Halorrhena pubescens, Lagenaria siceraria, Lepidium sativum, Mangifera indica, Medicago sativa, Myristica fragrans, Nigella sativa, Oryza sativa, Peganum harmala, Pennisetum glaucum, Picrorhiza kurroa, Piper betle, Prunus dulcis, Trifolium alexandrinum, Withania somnifera and Zea mays were found to be used for different indications in equines. As far as could be ascertained, there is no published literature on the use of plants for the treatment of ailments in equines. There are thousands $(\approx 250,000)$ of species of plants naturally available [40], with a low proportionate exploited for medicinal purposes. Further, $5-5 \%$ of the higher plants have been investigated for their active constituents against a wide range of infectious and non-infectious diseases of humans and animals 
(Pieters and Vlietinck [41]). Plants are primary source of natural products used by traditional healers in $80 \%$ of the developing societies [42]. There was about $40 \%$ repetition in the ethno-botanical preparations (EBPs) documented in the present study and those documented by others for other species of animals [28-32]). It has been reported previously that different parts of the same plant (leaves, fruits, flowers, seeds, seed kernels, latex, stem, grains, bulbs, tuber, roots, basal rosette, bark, thallus, shoots, wood, buds, aerial parts, branches, etc.) and variety of solvents used for their extraction diversify their usage ([43-46]). Variation in the doses and mode of preparation of remedies within and among different conditions has also been reported elsewhere [11,28,30,32,47]. The aspect of non-standardized doses in phytotherapy have been criticized, because of toxicity constraints, under dosing, and cost; however, cost can be reduced by proper standardization of doses [48,49]. The common adage that natural is synonymous with safe, has led to significant and widespread disease [50], and it is critical that those involved in equine husbandry and health care are aware of the potential dangers of herbal medicine. Therefore, researches on the standardization of doses regarding efficacy $v s$ safety should be carried out.

\section{Conclusions}

The current research suggests that EBPs have a crucial role in animal health and production in the study area. The current study revealed a diverse range of plants which is in practice to treat the prevalent ailments in equine population of Punjab. The pitfalls of TVPs found in this study were related with the improper diagnosis of diseases (some cases), non-standardization of dosages, mode of preparation and administration, and lack of understanding regarding importance of value addition (e.g., validation) to the existing practices, adverse/overdosing effects and documentation of indigenous knowledge. For example, traditional healers were not aware of the minimum essentials of parasite biology and strategic worm control practices. Fundamental issues in phytotherapy; however, are the dose, efficacy and safety left to an educated guess or is completely ignored. However, a handsome volume of the indigenous knowledge has been documented for the first time in the region in relation to the treatment of equines which provides a baseline for future scientific investigations in phytochemistry. The promising candidates of plant origin can be isolated through modern chemistry protocols and authenticated for their medical value after in vivo and in vitro experimentations.

\section{Competing interests}

We declare that none of the authors have competing interests.

\section{Authors' contributions}

Zl: designed the project for the Ph.D. research of KG who was actively involved in the field surveys and compilation of the data. MS provided the transport and consultation services for approaching the equines of the study area. MSS provided comments and suggestions during drafting and reporting of the data and wrote the draft of manuscript. Q was involved in writing and reviewing of manuscript. All authors approve the final submission of the manuscript.

\section{Acknowledgements}

The financial support of the Higher Education Commission, Islamabad for completion of this study is acknowledged. Authors would like to thank all the local veterinary healers of the study districts who participated for making this surveillance a success. Professional collaboration of The Brookes Hospital for Animals at the Faculty of Veterinary Sciences is highly appreciable for making field visits possible.

\section{Author details}

${ }^{1}$ Department of Parasitology, University of Agriculture, Faisalabad 38040 Punjab, Pakistan. ${ }^{2}$ Department of Veterinary Clinical Medicine and Surgery, University of Agriculture, Faisalabad 38040 Punjab, Pakistan. ${ }^{3}$ Brookes Hospital for Animals, Faculty of Veterinary Science, University of Agriculture, Faisalabad 38040 Punjab, Pakistan.

Received: 12 March 2013 Accepted: 22 September 2013

Published: 30 September 2013

\section{References}

1. Urquhart GM, Armour J, Duncan JL, Dunn AM, Jennings FW: Veterinary Parasitology. 2nd edition. UK: Blackwell Science; 2007.

2. Goraya K, lqbal Z, Sajid MS, Muhammad G: Frequency distribution of equine diseases in three metropolises of the upper Punjab, Pakistan. Int J Agric Biol 2013, 00:000-000.

3. Lans C, Turner N, Brauer G, Lourenco G, Georges K: Ethnoveterinary medicines used for horses in Trinidad and in British Columbia, Canada. J Ethnobiol Ethnomed 2006, 2:31.

4. Cox PA: Will tribal knowledge survive the millennium? Science 2000, 287:44-45.

5. Ole-Miaron JO: Ethnoveterinary practices of the Loitokitock Maasai: impact on the environment. Vet J 1997, 17:159-167.

6. Lans C: Creole remedies: case studies of ethnoveterinary medicine in Trinidad and Tobago. PhD Thesis. Netherlands: Wageningen University; 2001.

7. Akhtar MS, lqbal Z, Khan MN, Lateef M: Anthelmintic activity of medicinal plants with particular reference to their use in animals in the Indo-Pakistan subcontinent. Small Rumin Res 2000, 38:99-107.

8. labal Z, Lateef M, Jabbar A, Muhammad G, Khan MN: Anthelmintic activity of Calotropis procera (Ait.) Ait. F. flowers in sheep. J Ethnopharmacol 2005, 102:256-261.

9. Iqbal Z, Sarwar M, Jabbar A, Ahmad S, Nisa M, Sajid MS, Khan MN, Mufti KA, Yaseen M: Direct and indirect anthelmintic effects of condensed tannins in sheep. Vet Parasitol 2007, 144:125-131.

10. Lateef M, Iqbal Z, Sajid MS, Abbas RZ, Sindhu ZUD, Akhtar M, Khan MN, Awais MM, labal A, Ain QU: An account of botanical anthelmintics and methods used for their evaluation. Rev Vet Anim Sci 2013, 1:7-14.

11. Sindhu ZUD, Iqbal Z, Khan MN, Jonsson NN, Siddique M: Documentation of ethno - veterinary practices used for treatment of different ailments in selected a hilly area of Pakistan. Int J Agric Biol 2010, 12:353-358.

12. Sindhu ZUD, Shafiq-Ullah, Abbas RZ, lqbal Z, Hameed M: Inventory of ethno-veterinary practices used for the control of parasitic infections in district Jhang, Pakistan. Int J Agric Biol 2012, 14:922-928.

13. Anonymous: Pakistan livestock census. Pakistan, Islamabad: Agricultural Census Organization, Ministry of Economic Affairs and Statistics; 2006.

14. Dunn T: Rapid rural appraisal: a description of the methodology and its application in teaching and research at Charles Stuart University. Wagga Wagga Australia: Rural Society; 1992-1994.

15. Thrusfield M: Veterinary epidemiology. 3rd edition. Blackwell science; 2007:231-232.

16. Etkins NL: Anthropological methods in ethnopharmacology. J Ethnopharmacol 1993, 38:93-104. 
17. Ashfaq M, Ashfaq U: Evalauation of mosquitocidal activity of water extract of Moringa Oleifera seeds against Culex Quinuefasciatus (Diptera: Culicidae) in Pakistan. Pak Entomol 2012, 34:21-26.

18. Waller PJ, Bernes G, Thamsborg SM, Sukura A, Richter SH, Ingebrigtsen $K$, Hoglund J: Plants as de-worming agents of livestock in the Nordic countries: historical perspective, popular beliefs and prospects for the future. Acta Vet Scand 2001, 42:31-44.

19. Wang P, Su Z, Yuan W, Deng G, Li S: Phytochemical constituents and pharmacological activities of Eryngium L. (Apiaceae). Pharmaceutical Crops 2012, 3:99-120.

20. De Leeuw PN, McDermott JJ, Lebbie SHB: Monitoring of livestock health and production in sub - Saharan Africa. Prev Vet Med 1995, 25:195-212.

21. Bennet-Jenkins E, Bryant C: Novel sources of anthelmintics. Int J Parasitol 1996, 26:937-947.

22. Brokensha DW, Warren DM, Werner O: Indigenous knowledge systems and development. Lanham, MD: University Press of America; 1980.

23. IDS: Whose knowledge counts? IDS Bull 1979, 10:2.

24. Schultes RE: The kingdom of plants: medicines from the earth, Thomson, WAR. New York, NY: McGraw-Hill Book Co; 1978:208.

25. Nok AJ, Esievo AN, Longet I, Arowosafe S, Onyenekwe PC, Gimba CE, Kagbu JA: Trypanocidal potentials of Azadirachta indica: in vivo activity of leaf extract against Trypanosoma brucei. J Clin Biochem Nutr 1993, 15:113-118.

26. Nok AJ, Esievo KAN, Armbrose A, Isaac Al, Emmanuel GC, Solomon MO, James KA: Trypanocidal activity of an organotin compound (tri-n-butyltin oxide) toward Trypanosoma brucei. J Clin Biochem Nutr 1992, 13:81-85.

27. Nok AJ, Williams S: Allium sativum induced death of African Trypanosomes. Parasitol Res 1996, 82:634-637.

28. Dilshad SMR, Rehman N, lqbal Z, Muhammad G, lqbal A, Ahmed N: An inventory of the ethnoveterinary practices for reproductive disorders in cattle and buffaloes, Sargodha district of Pakistan. J Ethnopharmacol 2008, 117:393-402

29. Farooq Z, lqbal Z, Mushtaq S, Muhammad G, lqbal MZ, Arshad M: Ethnoveterinary practices for the treatment of parasitic diseases in livestock in Cholistan desert (Pakistan). J Ethnopharmacol 2008, 118:213-219.

30. Jabbar A, lqbal Z, Khan MN: In vitro anthelmintic activity of Trachyspermum ammi seeds. Phcog Mag 2006b, 2:126-129.

31. Khan MK, Sajid MS, Khan MN, Iqbal Z, lqbal MU: Bovine fasciolosis: prevalence, effects of treatment on productivity and cost benefit analysis in five districts of Punjab, Pakistan. Res Vet Sci 2009, 87:70-75.

32. Muhammad G, Khan MZ, Hussain MH, lqbal Z, Iqbal M, Athar M: Ethnoveterinary practices of owners of pneumatic-cart pulling camels in Faisalabad city (Pakistan). J Ethnopharmacol 2005, 97:241-246.

33. Aqel MB: Relaxant effect of the volatile oil of rosmarinus officinalis on tracheal smooth muscle. J Ethnopharmacol 1991, 33:57-62

34. Lanhers MC, Fleurentin J, Mortier F, Vinche A, Younos C: Anti-Inflammatory and analgesic effects of an aqueous extract of Harpagophytum procumbens. Planta Med 1992, 58:17-123.

35. Pearson W: Ethnoveterinary medicine: the science of botanicals in equine health and disease, Proceedings of the 2nd European equine health and nutrition congress, lelystad. The Netherlands; 2003:31-40.

36. Sommer H, Felbinger U, Pütz R, Reutershan R, Schaefer J: The effects of an herb mixture on horses with respiratory disease. Tierarztl Umsch 1986, 41:846-848.

37. Wagner I, Greim C, Laufer S, Heide L, Gleiter CH: Influence of willow bark extract cyclooxygenase activity and on tumor necrosis factor alpha or interleukin 1 beta release in vitro and ex vivo. Clin Pharmacol Ther 2003, 73:272-274.

38. Iwu M: Handbook of African medicinal plants. Boca Raton, FL: CRC Press; 1993.

39. Reichling J, Saller R: Herbal remedies in veterinary phytotherapy. Schweiz Arch Tierheilkd 2001, 43:395-403.

40. Borris RP: Natural products research: perspectives from a major pharmaceutical company. J Ethnopharmacol 1996, 51:29-38.

41. Pieters $L$, Vlietnick AJ: Bioguide isolation of pharmacologically active plant components, still a valuable strategy for the finding of new lead compounds. Journal of Ethnopharmacology 2005, 100:57-60.

42. Farnsworth NR, Akerele O, Bingel AS, Soejarto DD, Guo Z: Medicinal plants in therapy. Bull World Health Organ 1985, 63:965-981.

43. Giday M, Asfaw Z, Elmqvist T, Woldu Z: An ethnobotanical study of medicinal plants used by the Zay people in Ethiopia. J Ethnopharmacol 2003, 85:43-52.
44. Nfi AN, Mbanya JN, Ndi C, Kameni A, Vabi M, Pingpoh D, Yonkeu S, Moussa C: Ethnoveterinary medicine in the Northern Provinces of Cameroon. Vet Res Commun 2001, 25:71-76.

45. Ole-Miaron JO: The Maasai ethnodiagnostic skill of livestock diseases: a lead to traditional bioprospecting. J Ethnopharmacol 2003, 84:79-83.

46. Viegi L, Pieroni A, Guarrera PM, Vangelisti R: A review of plants used in folk veterinarymedicine in Italy as basis for a databank. J Ethnopharmacol 2003, 89:221-244.

47. Deeba F, Muhammad G, lqbal Z, Hussain I: Appraisal of ethno-veterinary practices used for different ailments in dairy animals in peri-urban areas of Faisalabad (Pakistan). Int J Agric Biol 2009, 11:535-541.

48. Bakhiet AO, Adam SEl: Therapeutic utility, constitutents and toxicity of some medicinal plants. Vet Human Toxicol 1995, 37:255-258.

49. Longuefosse $J$, Nossin E: Medical ethnobotany survey in Martinique. J Ethnopharmacol 1996, 53:117-120.

50. Pearson W: Pyrrolizidine Alkaloids in higher plants: hepatic veno-occlusive disease associated with chronic consumption. JNFMF 2000, 3:87-96.

\section{doi:10.1186/1746-4269-9-70}

Cite this article as: Goraya et al:: Diversity of flora used for the cure of equine diseases in selected peri-urban areas of Punjab, Pakistan. Journal of Ethnobiology and Ethnomedicine 2013 9:70.

\section{Submit your next manuscript to BioMed Central and take full advantage of:}

- Convenient online submission

- Thorough peer review

- No space constraints or color figure charges

- Immediate publication on acceptance

- Inclusion in PubMed, CAS, Scopus and Google Scholar

- Research which is freely available for redistribution
C Biomed Central 